\title{
EL SUICIDIO EN LA ANCIANIDAD EN ESPAÑA
}

\section{Gerardo Hernández Rodríguez}

Universidad de La Coruña

\section{INTRODUCCION}

Hay hechos y fenómenos sociales que cuando se producen provocan un impacto especial, una impresión y unas reacciones particulares en la opinión pública y en la sociedad en general. Uno de estos hechos es la muerte autoprovocada, y especialmente, aunque por razones muy diferentes, si se trata de suicidios en niños y jóvenes o en ancianos. La sociedad es particularmente sensible a determinadas situaciones y circunstancias.

En una publicación anterior ${ }^{1}$, ya fue analizado el fenómeno del suicidio de los ancianos en España desde el año 1970 hasta 1981, subdividiéndolo en dos períodos (1970-1976 y 1977-1981), empleando entonces, para diferentes aspectos estudiados, como edades iniciales de referencia los 60, 65 y 70 años de edad, respectivamente.

Dado que en las Estadísticas del Suicidio en España, del Instituto Nacional de Estadística, el último intervalo de edad que se considera para la especificación de la mortalidad por suicidio y lesiones autoinfligidas (Rúbricas E950E959 de la Nomenclatura Internacional Detallada de causas de muerte) es el de 60 y más años, se ha decidido a la hora de acometer este estudio que ahora

A. Arbelo Curbelo, G. Hernández Rodríguez y A. Arbelo lópez de Letona, Demografía sanitaria de la ancianidad, Ministerio de Sanidad y Consumo, Madrid, 1989. 
nos ocupa realizarlo partiendo también de dicha edad, aunque, como ya ha quedado manifestado en ocasiones precedentes ${ }^{2}$, la edad que más frecuentemente se emplea para fijar, a efectos demográficos y estadísticos, el comienzo de la ancianidad es la de los 65 años.

Al llegar aquí es preciso señalar tres puntos.

El primero, que el estudio se centrará en los años que van desde 1976, último de los incluidos en el primer bloque de años considerados en la Demografía Sanitaria de la Ancianidad, ya citada, hasta 1992, último del que se dispone de datos publicados por el Instituto Nacional de Estadística en la actualidad, aunque se detallará en las tablas correspondientes (tabla I) la mortalidad real por esta causa desde 1970 hasta 1991.

\section{TABLA I}

España. Ancianos fallecidos por suicidios y lesiones autoinflingidas, E950-E959

\begin{tabular}{|c|c|c|c|c|c|c|c|c|c|}
\hline \multirow[b]{2}{*}{ Grupos de edades } & \multicolumn{3}{|c|}{1970} & \multicolumn{3}{|c|}{1971} & \multicolumn{3}{|c|}{1972} \\
\hline & $V$ & $M$ & $T$ & $V$ & $M$ & $T$ & $V$ & $M$ & $T$ \\
\hline 60 a $64 .$. & 125 & 51 & 176 & 128 & 42 & 170 & 117 & 33 & 150 \\
\hline 65 a 69 & 120 & 40 & 160 & 111 & 39 & 150 & 112 & 40 & 152 \\
\hline 70 a $74 \ldots \ldots \ldots \ldots \ldots \ldots$ & 87 & 30 & 117 & 93 & 40 & 133 & 105 & 36 & 141 \\
\hline 75 a 79 & 55 & 27 & 82 & 53 & 29 & 82 & 61 & 24 & 85 \\
\hline 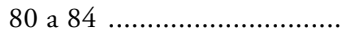 & 42 & 12 & 54 & 43 & 13 & 56 & 44 & 16 & 60 \\
\hline 85 y más ...... & 22 & 8 & 30 & 21 & 5 & 26 & 23 & 9 & 32 \\
\hline 60 y más & 451 & 168 & 619 & 449 & 168 & 617 & 462 & 158 & 620 \\
\hline Por 100 de todas edades ..... & 43 & 44 & 43 & 41 & 45 & 42 & 41 & 39 & 41 \\
\hline \multirow{3}{*}{$\begin{array}{c}\text { Todas edades ................ } \\
\text { Grupos de edades }\end{array}$} & 1.044 & 380 & 1.424 & 1.100 & 373 & 1.473 & 1.123 & 400 & 1.523 \\
\hline & & 1973 & & & 1974 & & & 1975 & \\
\hline & $V$ & $M$ & $T$ & $V$ & $M$ & $T$ & $V$ & $M$ & $T$ \\
\hline 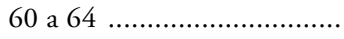 & 110 & 41 & 151 & 109 & 50 & 159 & 85 & 38 & 123 \\
\hline 65 a 69 & 125 & 50 & 175 & 101 & 41 & 142 & 118 & 41 & 159 \\
\hline 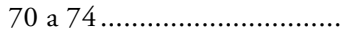 & 102 & 31 & 133 & 102 & 39 & 141 & 104 & 30 & 134 \\
\hline 75 a 79 & 53 & 26 & 79 & 67 & 28 & 95 & 57 & 23 & 80 \\
\hline 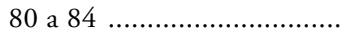 & 50 & 14 & 64 & 43 & 17 & 60 & 36 & 13 & 49 \\
\hline 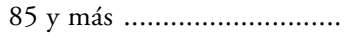 & 18 & 8 & 26 & 17 & 7 & 24 & 20 & 6 & 26 \\
\hline 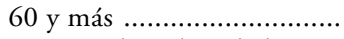 & 458 & 170 & 628 & 439 & 182 & 621 & 420 & 151 & 571 \\
\hline Por 100 de todas edades ..... & 44 & 41 & 43 & 45 & 46 & 45 & 42 & 43 & 42 \\
\hline Todas edades & 1.043 & 414 & 1.457 & 982 & 399 & 1.381 & 1.011 & 355 & 1.366 \\
\hline
\end{tabular}

${ }^{2}$ Ibidem, pp. 22-29. 
TABLA I (continuación)

España. Ancianos fallecidos por suicidios y lesiones autoinflingidas, E950-E959

\begin{tabular}{|c|c|c|c|c|c|c|c|c|c|}
\hline \multirow[b]{2}{*}{ Grupos de edades } & \multicolumn{3}{|c|}{1976} & \multicolumn{3}{|c|}{1977} & \multicolumn{3}{|c|}{1978} \\
\hline & $V$ & $M$ & $T$ & $V$ & $M$ & $T$ & $V$ & $M$ & $T$ \\
\hline 60 a 64 & 96 & 47 & 143 & 103 & 44 & 147 & 97 & 37 & 134 \\
\hline 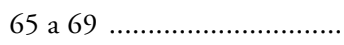 & 95 & 44 & 139 & 102 & 49 & 151 & 90 & 39 & 129 \\
\hline 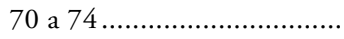 & 94 & 42 & 136 & 92 & 30 & 122 & 96 & 35 & 131 \\
\hline 75 a 79 & 75 & 27 & 102 & 66 & 28 & 94 & 65 & 31 & 96 \\
\hline 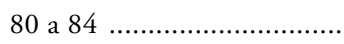 & 40 & 15 & 55 & 48 & 23 & 71 & 45 & 12 & 57 \\
\hline 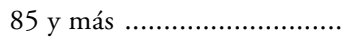 & 23 & 4 & 27 & 24 & 8 & 32 & 19 & 9 & 28 \\
\hline 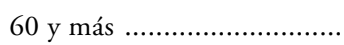 & . 423 & 179 & 602 & 435 & 182 & 617 & 412 & 163 & 575 \\
\hline Por 100 de todas edades ..... & 39 & 45 & 41 & 40 & 46 & 42 & 38 & 39 & 38 \\
\hline \multirow[t]{2}{*}{ 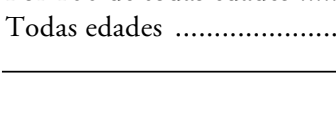 } & .073 & 401 & 1.474 & 1.089 & 397 & 1.486 & 1.094 & 413 & 1.507 \\
\hline & \multicolumn{3}{|c|}{1979} & \multicolumn{3}{|c|}{1980} & \multicolumn{3}{|c|}{1981} \\
\hline Grupos de edades & $V$ & $M$ & $T$ & $V$ & $M$ & $T$ & $V$ & $M$ & $T$ \\
\hline 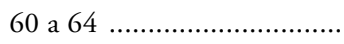 & 86 & 32 & 118 & 92 & 53 & 145 & 103 & 41 & 144 \\
\hline 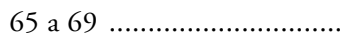 & 91 & 38 & 129 & 103 & 29 & 132 & 71 & 50 & 121 \\
\hline 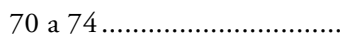 & 77 & 40 & 117 & 111 & 42 & 153 & 85 & 38 & 123 \\
\hline 75 a 79 & 98 & 23 & 121 & 76 & 38 & 114 & 87 & 37 & 124 \\
\hline 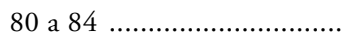 & 46 & 17 & 63 & 62 & 13 & 75 & 54 & 21 & 75 \\
\hline 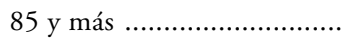 & 30 & 13 & 43 & 24 & 11 & 35 & 33 & 12 & 45 \\
\hline 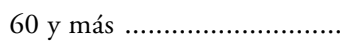 & 428 & 163 & 591 & 468 & 186 & 654 & 433 & 199 & 632 \\
\hline Por 100 de todas edades ..... & 37 & 42 & 39 & 38 & 45 & 40 & 34 & 44 & 37 \\
\hline \multirow{3}{*}{$\begin{array}{c}\text { Todas edades ................. } \\
\text { Grupos de edades }\end{array}$} & .1 .142 & 392 & 1.534 & 1.237 & 415 & 1.652 & 1.268 & 450 & 1.718 \\
\hline & \multicolumn{3}{|c|}{1982} & \multicolumn{3}{|c|}{1983} & \multicolumn{3}{|c|}{1984} \\
\hline & $V$ & $M$ & $T$ & $V$ & $M$ & $T$ & $V$ & $M$ & $T$ \\
\hline 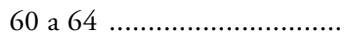 & 132 & 67 & 199 & 145 & 62 & 207 & 189 & 64 & 253 \\
\hline 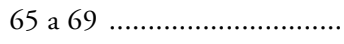 & 75 & 56 & 131 & 106 & 56 & 162 & 114 & 58 & 172 \\
\hline 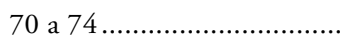 & . 103 & 43 & 146 & 121 & 67 & 188 & 143 & 64 & 207 \\
\hline 75 a 79 & . $\quad 102$ & 40 & 142 & 132 & 50 & 182 & 132 & 70 & 202 \\
\hline 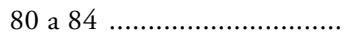 & 50 & 21 & 71 & 86 & 32 & 118 & 84 & 32 & 116 \\
\hline 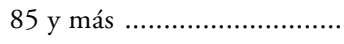 & 37 & 12 & 49 & 39 & 18 & 57 & 41 & 22 & 63 \\
\hline 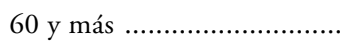 & . 499 & 239 & 738 & 629 & 285 & 914 & 703 & 310 & 1.013 \\
\hline Por 100 de todas edades ..... & 37 & 46 & 40 & 39 & 48 & 42 & 38 & 47 & 41 \\
\hline 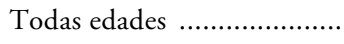 & $\cdot 1.337$ & 515 & 1.852 & 1.593 & 588 & 2.181 & 1.836 & 657 & 2.493 \\
\hline
\end{tabular}




\section{TABLA I (continuación)}

España. Ancianos fallecidos por suicidios y lesiones autoinflingidas, E950-E959

\begin{tabular}{|c|c|c|c|c|c|c|c|c|c|}
\hline \multirow[b]{2}{*}{ Grupos de edades } & \multicolumn{3}{|c|}{1985} & \multicolumn{3}{|c|}{1986} & \multicolumn{3}{|c|}{1987} \\
\hline & $V$ & $M$ & $T$ & $V$ & $M$ & $T$ & $V$ & $M$ & $T$ \\
\hline 60 a $64 .$. & 176 & 78 & 254 & 179 & 71 & 250 & 194 & 81 & 275 \\
\hline 65 a 69. & 110 & 71 & 181 & 137 & 71 & 208 & 162 & 78 & 240 \\
\hline 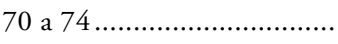 & 139 & 66 & 205 & 147 & 86 & 233 & 155 & 81 & 236 \\
\hline 75 a 79 & 137 & 69 & 206 & 149 & 48 & 197 & 156 & 60 & 216 \\
\hline 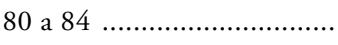 & 114 & 37 & 151 & 98 & 52 & 150 & 118 & 42 & 160 \\
\hline 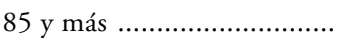 & 54 & 25 & 79 & 58 & 27 & 85 & 61 & 25 & 86 \\
\hline 60 y más. & 730 & 346 & 1.076 & 768 & 355 & 1.123 & 846 & 367 & 1.213 \\
\hline Por 100 de todas edades ..... & 40 & 51 & 43 & 38 & 49 & 41 & 42 & 47 & 43 \\
\hline \multirow{3}{*}{$\begin{array}{c}\text { Todas edades .................. } \\
\text { Grupos de edades }\end{array}$} & 1.829 & 685 & 2.514 & 2.032 & 723 & 2.755 & 2.017 & 787 & 2.804 \\
\hline & \multicolumn{3}{|c|}{1988} & \multicolumn{3}{|c|}{1989} & \multicolumn{3}{|c|}{1990} \\
\hline & $V$ & $M$ & $T$ & $V$ & $M$ & $T$ & $V$ & $M$ & $T$ \\
\hline 60 a 64. & 183 & 75 & 258 & 161 & 78 & 239 & 174 & 60 & 234 \\
\hline 65 a 69. & 154 & 86 & 240 & 180 & 82 & 262 & 170 & 84 & 254 \\
\hline 70 a $74 \ldots \ldots \ldots \ldots \ldots \ldots \ldots \ldots$ & 144 & 78 & 222 & 163 & 92 & 255 & 148 & 72 & 220 \\
\hline 75 a 79 & 163 & 82 & 245 & 152 & 65 & 217 & 177 & 71 & 248 \\
\hline 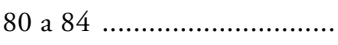 & 117 & 39 & 156 & 141 & 49 & 190 & 142 & 52 & 194 \\
\hline 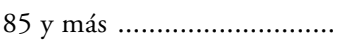 & 74 & 35 & 109 & 66 & 38 & 104 & 85 & 52 & 137 \\
\hline 60 y más. & 835 & 395 & 1.230 & 863 & 404 & 1.267 & 896 & 391 & 1.287 \\
\hline Por 100 de todas edades ..... & 39 & 49 & 42 & 39 & 52 & 42 & 42 & 49 & 44 \\
\hline \multirow{3}{*}{$\begin{array}{c}\text { Todas edades ................. } \\
\text { Grupos de edades }\end{array}$} & 2.150 & 810 & 2.960 & 2.213 & 774 & 2.987 & 2.135 & 804 & 2.939 \\
\hline & \multicolumn{5}{|c|}{1991} & \multicolumn{4}{|c|}{$1970 / 1991$} \\
\hline & $V$ & & $M$ & $T$ & & $V$ & $M$ & & $T$ \\
\hline 60 a 64 & 182 & & 69 & 251 & & 2.966 & 1.214 & & 4.180 \\
\hline 65 a 69 & 197 & & 76 & 273 & & 2.644 & 1.218 & & 3.862 \\
\hline 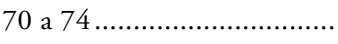 & 155 & & 91 & 246 & & 2.566 & 1.173 & & 3.739 \\
\hline 75 a 79 & 161 & & 79 & 240 & & 2.294 & 975 & & 3.269 \\
\hline 80 a $84 \ldots \ldots \ldots \ldots \ldots \ldots \ldots \ldots$ & 112 & & 55 & 167 & & 1.615 & 597 & & 2.212 \\
\hline 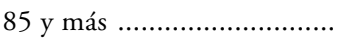 & 77 & & 38 & 115 & & 948 & 415 & & 1.363 \\
\hline 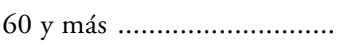 & 884 & & 408 & 1.292 & & 13.033 & 5.592 & & 18.625 \\
\hline Por 100 de todas edades ..... & 41 & & 53 & 44 & & 40 & 47 & & 42 \\
\hline 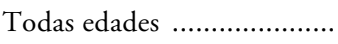 & 2.139 & & 777 & 2.917 & & 32.569 & 11.976 & & 44.545 \\
\hline
\end{tabular}


El segundo, que desde el año 1981 el Instituto Nacional de Estadística no emplea, para el cruce de las variables sexo y edad, la Nomenclatura Detallada, sino la Reducida, es decir, que sólo consta el número de ancianos y ancianas fallecidos por «Suicidio y lesiones autoinfligidas», en su totalidad y no pormenorizados, según el procedimiento empleado. Por consiguiente, en las tablas correspondientes a los métodos utilizados, según las diversas rúbricas, existen, para cada una de ellas, dos grupos. El primero corresponde a los fallecimientos reales, según los datos del Movimiento Natural de la Población, causas de muerte según las rúbricas de la citada Nomenclatura Internacional Detallada, desde el año 1970 hasta el año 1975, y el segundo corresponde a los suicidios consumados y a las tentativas, desde 1976 hasta 1992, a fin de simplificar las fuentes de referencias y de acuerdo con el plan trazado para la elaboración de este trabajo, según el procedimiento o sistema utilizado, a partir de los datos que figuran en las Estadisticas del Suicidio en España.

Y el tercero, que, por tanto, aquí se tendrán en cuenta no sólo los fallecimientos reales por suicidio y lesiones autoinfligidas, sino también las tentativas, especificándolo, para mejor comprensión y claridad expositiva, cuando así proceda.

Así, pues, analizaremos las diferentes causas de muerte y los procedimientos empleados. Asimismo, se verán el estado civil, la profesión y las razones existentes, cuando son conocidas, que han llevado a los suicidas, tanto en su grado de tentativa como de consumación, a tomar tal decisión.

La estadística del suicidio se realiza, ininterrumpidamente, desde principios de siglo, en cumplimiento de lo dispuesto en la Real Orden de 8 de septiembre de 1906.

El Instituto Nacional de Estadística distribuye, a través de sus Delegaciones Provinciales, a todos los Juzgados de Instrucción los boletines de suicidio, que deben ser cumplimentados por los antedichos Juzgados. Se recopila la información tanto de los suicidios consumados como de las tentativas, ya que lo que se pretende estudiar por parte del mencionado organismo es el acto del suicidio, con todas sus consecuencias de tipo social que puedan tener interés, como son, entre otros, el nivel de estudios, la situación profesional, la edad, el sexo, etc., independientemente del resultado.

Por otro lado, la investigación del fenómeno del suicidio tropieza con dificultades que derivan, en primer lugar, de la dificultad de establecer la certidumbre de que haya existido, efectivamente, una decisión de terminar con la propia vida, de determinar las verdaderas motivaciones que inducen a tal decisión, y, en segundo término, la posible pérdida de información de aquellas tentativas de suicidio que, por no haber requerido de internamiento hospitalario, no han dado lugar a la apertura de procedimiento judicial alguno.

La mecánica que se sigue posteriormente es la siguiente: los Juzgados remiten mensualmente a las Delegaciones Provinciales del Instituto Nacional de Estadística los diferentes boletines a razón de uno por cada supuesto, desde donde, una vez depurados, se envían al Servicio de Estadísticas Judiciales para 
su codificación y grabación; siendo elaboradas las tablas correspondientes para su publicación anual desde 1986, ya que anteriormente dicha publicación era quinquenal.

No obstante, en este punto es preciso destacar la diferencia existente entre los datos que figuran en las Estadísticas de Suicidios en España y en el Anuario Estadístico de España, en los que se incluyen las tentativas y los consumados, y los detallados en el Movimiento Natural de la Población. Defunciones según la causa de muerte. Las cifras obtenidas de esta última publicación son, paradójica e incomprensiblemente y como se podrá apreciar en su momento, superiores a las de aquéllas.

\section{SUICIDIOS Y LESIONES AUTOINFLIGIDAS}

El subgrupo de rúbricas E950-E959 — Suicidio y lesiones autoinfligidasque examinamos recogió la cifra de 18.625 ancianos muertos desde 1970 hasta 1991 , que equivale a un promedio anual de 847 . Su peso en el total de las defunciones de todas las edades por la misma causa es del 41,8 por 100 .

La distribución según sexo hombre/mujer fue, en cifras absolutas, $13.033 / 5.592$ (70/30 por 100$)$, lo que señala en forma clara la hipermortalidad masculina.

La repartición en los grupos quinquenales de edades en que la estadística oficial recoge las defunciones nos muestra cómo, en general, el número de ancianos muertos disminuye conforme es mayor la edad, hecho que sucede en ambos sexos, aunque con ligeras fluctuaciones.

Sin embargo, hay que señalar tres hechos ciertos. El primero de ellos es que el número total de ancianos y ancianas fallecidos por suicidio va aumentando progresivamente, en términos absolutos, a partir de 1982 . El promedio anual de suicidios en la ancianidad fue de 615 entre 1970 y 1982, y de 899 entre 1983 y 1992. Y teniendo como base 100 en 1970, con 643 suicidios de personas con edades superiores a los 60 años, se pasa a una relación de 102,3 en 1976, con 658 fallecimientos por esta causa y en estas edades, y a 169,4 en 1991. Los suicidios y tentativas en la ancianidad suponen en 1970 el 39 por 100 de los suicidios y tentativas en todas las edades, el 38 por 100 en 1976 y el 41 por 100 en 1992.

El segundo, que a partir de 1980 se produce un aumento en los suicidios en los intervalos de edad de 70 a 74 y de 75 a 79 años con respecto a los intervalos anteriores, aunque luego vuelve a remitir el número de fallecidos por esta causa en las edades de la ancianidad.

$\mathrm{Y}$ el tercero es que, pese a que los hombres ancianos alcanzan unas cifras más elevadas de suicidios que las mujeres ancianas, éstas, en un número proporcionalmente alto de años, llegan a porcentajes, en relación con el número total de suicidas femeninas de todas las edades, muy superiores a los de los hombres, aproximándose en algunos casos, y superándolo en otros (años 1985, 
1986, 1988, 1989, 1990, 1991), al 50 por 100 del total de suicidios femeninos. En cambio, el coeficiente de 0,13 por 1.000 ancianos del mismo grupo de edad se mantiene casi en el mismo valor. La evolución de la mortalidad es de permanencia de los valores en torno al 0,13, a expensas del sexo masculino.

Sin duda, los suicidios y lesiones autoinfligidas del anciano son llevadas a cabo en circunstancias especiales de su vida, alteración de su salud psicofísica y las de su entorno familiar y social, que en simples causas o concausas determinan las diversas clases de lesiones que ponen fin a su existencia.

En la actualidad habría que añadir a estas tradicionales causas familiares y sociales las coadyuvantes de los factores negativos de la socialización de nuestro entorno más inmediato, como podremos ver más adelante cuando nos refiramos a las causas o razones del suicidio en la ancianidad.

Para mejor ilustración del fenómeno objeto de análisis en este epígrafe se incluye en la tabla II el número de suicidios y tentativas acaecidos en España desde 1970 hasta 1992, con los respectivos coeficientes por 100.000 habitantes, y en la tabla III el detalle de los fallecimientos por suicidios y lesiones autoinfligidas en grupos quinquenales y con especificación, a efectos comparativos, de la relación entre sexos y el conjunto de óbitos producidos en ambos, desde 1970 a 1991.

Los fallecidos por suicidios y lesiones autoinfligidas en España en edades de la preancianidad ( 60 a 64 años) y ancianidad (65 y más años) fueron en total, como ya ha quedado expuesto anteriormente, 18.625 , el 41,8 por 100 del total de todas las edades.

Los datos contenidos en estas tablas ponen de manifiesto que el promedio anual de suicidios ha sido de 2.024; que se han quitado la vida voluntariamente, en un intervalo de veintidós años, un 41,8 por 100 de las personas mayores de 60 años, respecto del total de los suicidas; que, en relación con el mismo grupo de personas, un porcentaje del 3,9 por 100 eran niños o adolescentes, el 5,9 por 100 eran jóvenes de 20 a 24 años, y que, del total, un 73,1 por 100 eran hombres y el 26,9 por 100 eran mujeres.

De los mayores, de las personas de 60 y más años, entre los que el promedio anual de suicidios es de 847 , un 69,9 por 100 corresponde a los varones y el 30,1 a las mujeres. Con ello se comprueba que el promedio ha aumentado en 209 suicidios anuales, respecto de lo ocurrido en 1987, y que el porcentaje de suicidios femeninos en la ancianidad también se ha incrementado desde entonces en un 1,0 por 100. Del resto de los intervalos decenales, es el porcentaje más alto (18,5 por 100) el de los 50 a los 59 años. 


\section{TABLA II}

Suicidios y tentativas de suicidio. España 1970/1992

\begin{tabular}{|c|c|c|c|c|c|}
\hline Años & Consumados & Tentativas & No consta & Total & $\begin{array}{c}\text { Coeficiente } \\
\text { por } 100.000 \\
\text { habitantes }\end{array}$ \\
\hline 1970 ................ & 1.424 & 205 & - & 1.629 & 4,8 \\
\hline $1971 \ldots \ldots \ldots \ldots \ldots$ & 1.473 & 273 & - & 1.746 & 5,1 \\
\hline 1972 ................. & 1.523 & 316 & - & 1.839 & 5,3 \\
\hline $1973 \ldots \ldots \ldots \ldots \ldots$ & 1.457 & 313 & - & 1.770 & 5,1 \\
\hline $1974 \ldots \ldots \ldots \ldots \ldots$ & 1.381 & 336 & - & 1.717 & 4,9 \\
\hline 1975 ................. & 1.442 & 305 & - & 1.747 & 4,9 \\
\hline $1976 \ldots \ldots \ldots \ldots \ldots$ & 1.451 & 295 & - & 1.746 & 4,8 \\
\hline 1977 ................ & 1.378 & 245 & - & 1.623 & 4,4 \\
\hline 1978 ................ & 1.253 & 265 & - & 1.518 & 4,1 \\
\hline 1979 .................. & 1.225 & 148 & - & 1.373 & 3,7 \\
\hline 1980 .................. & 1.382 & 176 & - & 1.558 & 4,2 \\
\hline $1981 \ldots \ldots \ldots \ldots \ldots$ & 1.297 & 138 & - & 1.435 & 3,8 \\
\hline 1982 ................ & 1.266 & 113 & 84 & 1.463 & 3,9 \\
\hline 1983 ................. & 1.606 & 140 & 95 & 1.841 & 4,8 \\
\hline 1984 .................. & 1.935 & 188 & 27 & 2.150 & 5,6 \\
\hline 1985 ................. & 1.908 & 145 & 89 & 2.142 & 5,6 \\
\hline $1986 \ldots \ldots \ldots \ldots . . . . . . . .$. & 1.848 & 99 & 113 & 2.060 & 5,4 \\
\hline 1987 ................ & 1.868 & 132 & 118 & 2.118 & 5,5 \\
\hline $1988 \ldots \ldots \ldots \ldots \ldots$ & 2.137 & 148 & 146 & 2.431 & 6,3 \\
\hline 1989 ................. & 1.885 & 142 & 134 & 2.161 & 5,5 \\
\hline 1990 ................. & 2.012 & 185 & 102 & 2.299 & 5,9 \\
\hline 1991 ……............ & 2.243 & 224 & 132 & 2.599 & 6,7 \\
\hline $1992 * \ldots \ldots \ldots \ldots \ldots$ & 2.070 & 190 & 112 & 2.372 & 6,1 \\
\hline TOTAL ............ & 37.464 & 4.721 & 1.152 & 43.337 & \\
\hline
\end{tabular}

* Cifras provisionales.

FUENTE: Anuario Estadístico de España. Varios años. INE. Madrid. 


\section{TABLA III}

España. Fallecidos por suicidios y lesiones autoinflingidas. E950-E959. Años 1970/1991

\begin{tabular}{|c|c|c|c|c|}
\hline Grupos de edades & $V$ & $M$ & $T$ & Por 100 del total \\
\hline Menos de 10 años .......... & 11 & 0 & 11 & 0,0 \\
\hline 10 a 14 años & 252 & 57 & 308 & 0,7 \\
\hline 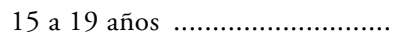 & 1.101 & 334 & 1.435 & 3,2 \\
\hline 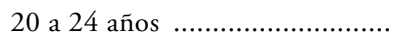 & 2.130 & 542 & 2.645 & 5,9 \\
\hline 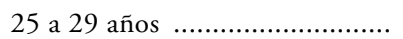 & 2.035 & 537 & 2.572 & 5,8 \\
\hline 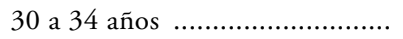 & 1.753 & 547 & 2.300 & 5,2 \\
\hline 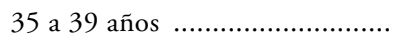 & 1.857 & 578 & 2.435 & 5,5 \\
\hline 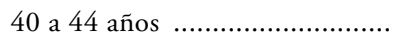 & 1.973 & 637 & 2.610 & 5,9 \\
\hline 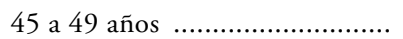 & 2.434 & 811 & 3.245 & 7,3 \\
\hline 50 a 54 años & 2.893 & 1.123 & 4.016 & 9,0 \\
\hline 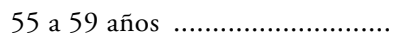 & 3.042 & 1.195 & 4.237 & 9,5 \\
\hline 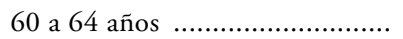 & 2.966 & 1.214 & 4.180 & 9,4 \\
\hline 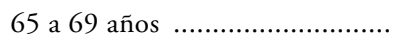 & 2.644 & 1.218 & 3.862 & 8,7 \\
\hline 70 a 74 años & 2.566 & 1.173 & 3.739 & 8,4 \\
\hline 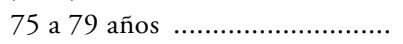 & 2.294 & 975 & 3.269 & 7,3 \\
\hline 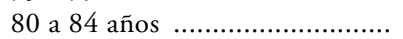 & 1.615 & 597 & 2.212 & 5,0 \\
\hline 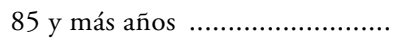 & 948 & 415 & 1.363 & 3,0 \\
\hline 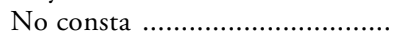 & 82 & 23 & 105 & 0,2 \\
\hline 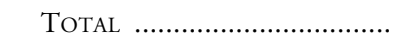 & 32.569 & 11.976 & 44.545 & 100,0 \\
\hline Promedio anual .... & 1.480 & 544 & 2.024 & \\
\hline 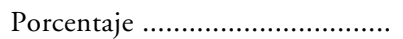 & 73,1 & 26,9 & & \\
\hline
\end{tabular}

\section{ESTADO CIVIL}

$\mathrm{Al}$ analizar los datos correspondientes a los suicidas ancianos en grado de tentativa y de consumación (tablas IV y V), siempre siendo mayor el número de hombres que el de mujeres, de aquéllos, el mayor porcentaje (56,8 por 100) corresponde a los casados, mientras que el de éstas (46,0 por 100) corresponde a las viudas.

En relación con el total de la población, del total de los suicidas viudos, el 87,9 por 100 eran ancianos, como lo eran también el 42,0 por 100 de los casados, el 21,8 por 100 de los divorciados y el 15,6 por 100 de los solteros. 


\section{TABLA IV}

Suicidios consumados y tentativas en la ancianidad (mayores de 60 años) por sexo y estado civil. Años 1976-1992

(Cifras absolutas)

\begin{tabular}{|c|c|c|c|c|c|}
\hline \multirow[b]{2}{*}{ Estado civil } & \multicolumn{3}{|c|}{ Sexo } & \multirow{2}{*}{$\begin{array}{c}\text { Total } \\
\text { más } 60 \text { años }\end{array}$} & \multirow{2}{*}{$\begin{array}{c}\text { Total } \\
\text { población }\end{array}$} \\
\hline & Hombres & Mujeres & No consta & & \\
\hline Solteros & 1.132 & 465 & 3 & 1.600 & 10.282 \\
\hline 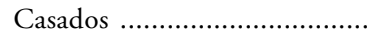 & 5.148 & 1.486 & 10 & 6.644 & 15.816 \\
\hline Viudos & 2.381 & 1.818 & 7 & 4.206 & 4.787 \\
\hline 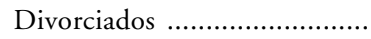 & 98 & 28 & 1 & 127 & 583 \\
\hline 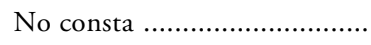 & 307 & 151 & 5 & 463 & 1.431 \\
\hline Total ancianos. & 9.066 & 3.948 & 26 & 13.040 & \\
\hline 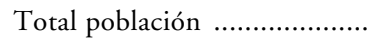 & 23.440 & 9.392 & 67 & & 32.899 \\
\hline
\end{tabular}

\section{TABLA V}

Suicidios consumados y tentativas en la ancianidad (mayores de 60 años) por sexo y estado civil. Años 1976-1992

(Porcentajes)

\begin{tabular}{|c|c|c|c|c|c|}
\hline \multirow[b]{2}{*}{ Estado civil } & \multicolumn{3}{|c|}{ Sexo } & \multirow{2}{*}{$\begin{array}{c}\text { Total } \\
\text { más } 60 \text { años }\end{array}$} & \multirow{2}{*}{$\begin{array}{c}\text { Total } \\
\text { población }\end{array}$} \\
\hline & Hombres & Mujeres & No consta & & \\
\hline Solteros ..................... & 12,5 & 11,8 & 11,5 & 12,3 & 31,2 \\
\hline 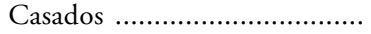 & 56,7 & 37,6 & 38,5 & 51,0 & 48,0 \\
\hline Viudos & 26,3 & 46,1 & 26,9 & 32,3 & 14,6 \\
\hline 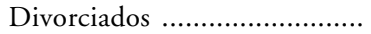 & 1,1 & 0,7 & 3,9 & 1,0 & 1,8 \\
\hline 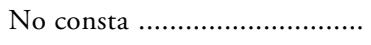 & 3,4 & 3,8 & 19,2 & 3,6 & 4,4 \\
\hline 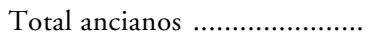 & 100,0 & 100,0 & 100,0 & 100,0 & 100,0 \\
\hline Total población ........................... & 71,3 & 28,6 & 0,2 & & 100,0 \\
\hline
\end{tabular}

\section{CAUSAS DE MUERTE Y PROCEDIMIENTOS EMPLEADOS}

De las diez rúbricas que integran este grupo y de las que, por las razones ya manifestadas anteriormente, hemos hecho dos subdivisiones: fallecimientos reales desde 1970 hasta 1975 y tentativas y suicidios consumados desde 1981 hasta 1992, ambas referidas a las edades de la ancianidad, las que mayor 


\section{TABLA VI}

España. Ancianos fallecidos por suicidios y lesiones autoinflingidas según las diversas rúbricas. E950-E959

(Años 1970/1975)

\begin{tabular}{|c|c|c|c|c|c|c|c|c|c|}
\hline \multirow[b]{2}{*}{ Años } & \multicolumn{3}{|c|}{$\begin{array}{c}\text { E950 } \\
\text { Envenenamiento } \\
\text { por sustancias } \\
\text { sólidas o líquidas }\end{array}$} & \multicolumn{3}{|c|}{$\begin{array}{c}\text { E951 } \\
\text { Envenenamiento } \\
\text { por gas de uso } \\
\text { doméstico }\end{array}$} & \multicolumn{3}{|c|}{$\begin{array}{c}\text { E952 } \\
\text { Envenenamiento } \\
\text { mediante otros } \\
\text { gases }\end{array}$} \\
\hline & $V$ & $M$ & $T$ & $V$ & $M$ & $T$ & $V$ & $M$ & $T$ \\
\hline 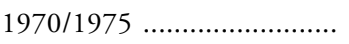 & 70 & 81 & 151 & 4 & 10 & 14 & 2 & 3 & 5 \\
\hline \multirow[t]{3}{*}{ Promedio anual ..................... } & 12 & 13 & 25 & 0,6 & 2 & 2 & 0,3 & 0,5 & 0,8 \\
\hline & \multicolumn{3}{|c|}{$\begin{array}{c}E 953 \\
\text { Por ahorcamiento, } \\
\text { estrangulación } \\
\text { y sofocación }\end{array}$} & \multicolumn{3}{|c|}{$\begin{array}{c}\text { E954 } \\
\text { Por sumersión } \\
\text { (ahogamiento) }\end{array}$} & \multicolumn{3}{|c|}{$\begin{array}{c}\text { E955 } \\
\text { Por armas } \\
\text { y explosivos }\end{array}$} \\
\hline & $V$ & $M$ & $T$ & $V$ & $M$ & $T$ & $V$ & $M$ & $T$ \\
\hline 1970/1975 .......................... & 1.484 & 319 & 1.803 & 394 & 283 & 677 & 128 & 3 & 131 \\
\hline \multirow[t]{3}{*}{ Promedio anual ....................... } & 247 & 53 & 300 & 66 & 47 & 113 & 21 & 0,5 & 22 \\
\hline & \multicolumn{3}{|c|}{$\begin{array}{c}\text { E956 } \\
\text { Por instrumento } \\
\text { cortante } \\
\text { o punzante }\end{array}$} & \multicolumn{3}{|c|}{$\begin{array}{c}\text { E957 } \\
\text { Por precipitación } \\
\text { de un lugar } \\
\text { elevado }\end{array}$} & \multicolumn{3}{|c|}{$\begin{array}{c}\text { E958 } \\
\text { Por otros } \\
\text { procedimientos } \\
\text { y no especificados }\end{array}$} \\
\hline & $V$ & $M$ & $T$ & $V$ & $M$ & $T$ & $V$ & $M$ & $T$ \\
\hline $1970 / 1975$........... & 80 & 25 & 105 & 299 & 222 & 521 & 217 & 50 & 267 \\
\hline \multirow[t]{3}{*}{ Promedio anual .................... } & 13 & 4 & 17 & 50 & 37 & 87 & 36 & 8 & 44 \\
\hline & \multicolumn{5}{|c|}{$\begin{array}{c}\text { E959 } \\
\text { Efectos tardios de lesiones } \\
\text { autoinflingidas }\end{array}$} & \multicolumn{4}{|c|}{$\begin{array}{l}\text { E950-E959 } \\
\text { Todas las causas }\end{array}$} \\
\hline & $V$ & & $M$ & $T$ & & $V$ & $M$ & & $T$ \\
\hline 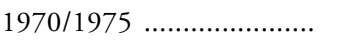 & 1 & & 0 & 1 & & 2.679 & 997 & & 3.676 \\
\hline Promedio anual ................... & 0,2 & & 0 & 0,2 & & 447 & 166 & & 613 \\
\hline
\end{tabular}

número de casos registran — referidas a la segunda subdivisión-, ordenadas de mayor a menor, sus valores del intervalo de tiempo analizado son: E953 - Suicidio y lesión autoinfligida por ahorcamiento, estrangulación y sofocación-, con 5.840 casos, cerca de la mitad de los suicidas mayores de 60 años $(44,8$ por 100$)$ y el 40,6 por 100 de todos los que emplearon este proce- 


\section{TABLA VII}

España. Suicidios (consumados y tentativas) en ancianos según las diversas rúbricas. E950-E959

(Años 1976/1992)

\begin{tabular}{|c|c|c|c|c|c|c|c|c|c|}
\hline \multirow[b]{2}{*}{ Años } & \multicolumn{3}{|c|}{$\begin{array}{c}\text { E950 } \\
\text { Envenenamiento } \\
\text { por sustancias } \\
\text { sólidas o líquidas }\end{array}$} & \multicolumn{3}{|c|}{$\begin{array}{l}\text { E951 } \\
\text { Envenenamiento } \\
\text { por gas de uso } \\
\text { doméstico }\end{array}$} & \multicolumn{3}{|c|}{$\begin{array}{c}\text { E952 } \\
\text { Envenenamiento } \\
\text { mediante otros } \\
\text { gases }\end{array}$} \\
\hline & $V$ & $M$ & $T$ & $V$ & $M$ & $T$ & $V$ & $M$ & $T$ \\
\hline $1976 / 1992$ & 305 & 324 & 629 & 28 & 14 & 42 & 32 & 7 & 39 \\
\hline \multirow[t]{3}{*}{ 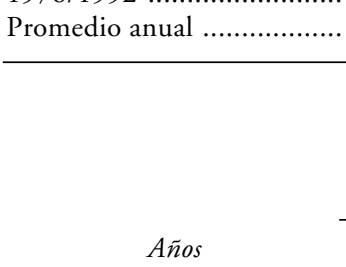 } & 18 & 19 & 37 & 2 & 1 & 2 & 2 & 0,4 & 2 \\
\hline & \multicolumn{3}{|c|}{$\begin{array}{c}\text { E953 } \\
\text { Por ahorcamiento, } \\
\text { estrangulación } \\
\text { y sofocación }\end{array}$} & \multicolumn{3}{|c|}{$\begin{array}{c}\text { E954 } \\
\text { Por sumersión } \\
\text { (ahogamiento) }\end{array}$} & \multicolumn{3}{|c|}{$\begin{array}{c}\text { E955 } \\
\text { Por armas } \\
\text { y explosivos }\end{array}$} \\
\hline & $V$ & $M$ & $T$ & $V$ & $M$ & $T$ & $V$ & $M$ & $T$ \\
\hline $1976 / 1992$. & 4.685 & 1.154 & 5.840 & 1.014 & 832 & 1.846 & 645 & 8 & 653 \\
\hline \multirow[t]{3}{*}{ Promedio anual } & 276 & & 344 & 60 & 49 & 109 & 38 & 0,4 & 38 \\
\hline & \multicolumn{3}{|c|}{$\begin{array}{c}\text { E956 } \\
\text { Por instrumento } \\
\text { cortante } \\
\text { o punzante }\end{array}$} & \multicolumn{3}{|c|}{$\begin{array}{c}\text { E957 } \\
\text { Por precipitación } \\
\text { de un lugar } \\
\text { elevado }\end{array}$} & \multicolumn{3}{|c|}{$\begin{array}{c}\text { E958 } \\
\text { Por otros } \\
\text { procedimientos } \\
\text { y no especificados }\end{array}$} \\
\hline & $V$ & $M$ & $T$ & $V$ & $M$ & $T$ & $V$ & $M$ & $T$ \\
\hline $1976 / 1992 \ldots \ldots \ldots$. & 247 & 106 & 353 & 1.477 & 1.259 & 2.736 & 541 & 198 & 739 \\
\hline Promedio anual ...................... & 15 & 6 & 21 & 87 & 74 & 161 & 32 & 12 & 44 \\
\hline
\end{tabular}

\section{E959}

Efectos tardios de lesiones autoinflingidas
E950-E959

Todas las causas

\begin{tabular}{|c|c|c|c|c|c|c|}
\hline Años & $V$ & $M$ & $T$ & $V$ & $M$ & $T$ \\
\hline $1976 / 1992$ & 0 & 0 & 0 & 9.077 & 3.963 & 13.040 \\
\hline Promedio anual .................. & 0 & 0 & 0 & 534 & 233 & 767 \\
\hline
\end{tabular}

dimiento; E957 — Por precipitación de un lugar elevado-, 2.736 muertes y tentativas $(21,0$ por 100$)$ y el 48,4 por 100 de quienes emplearon este sistema; E954 - Por sumersión (ahogamiento)—, 1.846 (14,2 por 100 de los ancianos y 60,5 por 100 del total de los ahogados); E958 - Por otros procedimientos y los no especificados (entre los que se halla el de arrojarse al paso de vehículos)—, 739 (5,7 por 100); E955 — Por armas de fuego y explosivos—, 653 


\section{TABLA VIII}

Suicidios consumados y tentativas en la ancianidad (mayores de 60 años) por sexo y procedimiento. Años 1976-1992

(Porcentajes)

\begin{tabular}{|c|c|c|c|c|c|}
\hline \multirow[b]{2}{*}{ Procedimiento } & \multicolumn{3}{|c|}{ Sexo } & \multirow{2}{*}{$\begin{array}{l}\text { Total } \\
\text { más } 60 \text { años }\end{array}$} & \multirow{2}{*}{$\begin{array}{c}\text { Total } \\
\text { población }\end{array}$} \\
\hline & Hombres & Mujeres & No consta & & \\
\hline Arma de fuego . ......................... & 7,1 & 0,2 & 3,9 & 5,0 & 8,2 \\
\hline Arma blanca .............................. & 2,7 & 2,7 & 3,9 & 2,7 & 3,3 \\
\hline Sumersión ............................... & 11,2 & 21,0 & 7,7 & 14,2 & 9,3 \\
\hline Envenenamiento (sustancias & & & & & \\
\hline sólidas, líquidas y gases) .... & 4,0 & 8,7 & 0,0 & 5,4 & 10,3 \\
\hline Axifisia y suspensión ............. & 51,6 & 29,1 & 34,6 & 44,8 & 43,7 \\
\hline Precipitándose desde altura $\ldots$ & 16,2 & 31,8 & 34,6 & 21,0 & 17,2 \\
\hline Arrojándose al paso de vehículo & 4,9 & 3,5 & 3,9 & 4,5 & 4,7 \\
\hline Otros procedimientos ............ & 1,1 & 1,5 & 3,9 & 1,2 & 1,8 \\
\hline No consta .............................. & 1,1 & 1,5 & 7,7 & 1,3 & 1,5 \\
\hline TOTAL ….............................. & 100,0 & 100,0 & 100,0 & 100,0 & 100,0 \\
\hline
\end{tabular}

(5,0 por 100); E950 - Envenenamiento por sustancias sólidas o líquidas-, 629 (4,8 por 100); E956 - Por instrumentos cortantes y punzantes-, 353 (2,7 por 100); E951 —Envenenamiento por gases de uso doméstico-, 42 (0,3 por 100$)$, y el noveno lugar a E952 - Envenenamiento mediante otros gases-, 39 (0,3 por 100).

No obstante sus bajas cifras, merece que nos detengamos en la rúbrica E951 - Envenenamiento mediante gas para uso doméstico-, en razón de poder tratarse de intoxicaciones involuntarias. El envenenamiento de muchos ancianos por gas ciudad, gas natural, gas butano o procedente de carbón mal quemado es motivado por olvido de comprobar varias veces al día, y al acostarse, si las llaves de paso del gas están cerradas, o quedarse dormidos por sueño o sopor sobre la mesa, estufa de gas o brasero (donde aún se utilice), o tener el baño con calentador de llama, a todo lo cual contribuye la pérdida del olfato en el anciano. Las normas preventivas para evitar estos envenenamientos o intoxicaciones serían: no usar brasero de carbón, chimeneas o lumbres bajas; no olvidar las instrucciones de la compañía del gas y de sus empleados en las revisiones periódicas de llaves, conducciones y quemadores, y control del cierre de llaves del gas varias veces al día.

Por lo que se refiere a los procedimientos empleados, merece la pena prestar una cierta atención al hecho diferenciador de sistemas «masculinos» $\mathrm{y}$ «femeninos».

El arma de fuego es empleada mayoritariamente por los hombres $(98,6$ por 
$100)$, así como el ahorcamiento $(80,1$ por 100$)$ y la precipitación al paso de vehículos (76,1 por 100). El envenenamiento lo utilizaron en el 51,5 por 100 de los casos las mujeres ancianas. Y del total de éstas, el 31,8 por 100 se precipitó desde una altura.

Respecto del total, los ancianos eligen, preferentemente, procedimientos definitivos, como lo demuestra el hecho de que un 40,6 por 100 de quienes acudieron al ahorcamiento fueran personas mayores de 60 años, un 48,4 por 100 de los que se precipitaron desde una altura y un 60,1 por 100 de los que se ahogaron o lo intentaron.

\section{PROFESIONES}

En la ancianidad, al tratar de verificar la profesión de quienes se han suicidado o lo han intentado, debido muy probablemente a que la mayoría ya están jubilados, la proporción más alta $(81,7$ por 100$)$ figura como que «No consta o no está bien especificado" (tablas IX y X). De entre aquellos a los que se les

\section{TABLA IX}

Suicidios consumados y tentativas en la ancianidad (mayores de 60 años) por sexo y profesiones. Años 1976-1992

(Cifras absolutas)

\begin{tabular}{|c|c|c|c|c|c|}
\hline \multirow[b]{2}{*}{ Profesiones } & \multicolumn{3}{|c|}{ Sexo } & \multirow{2}{*}{$\begin{array}{l}\text { Total } \\
\text { más } 60 \text { años }\end{array}$} & \multirow{2}{*}{$\begin{array}{c}\text { Total } \\
\text { población }\end{array}$} \\
\hline & Hombres & Mujeres & No consta & & \\
\hline $\begin{array}{l}\text { Profesionales, técnicos y asimi- } \\
\text { lados }\end{array}$ & 89 & 15 & 0 & 104 & 652 \\
\hline $\begin{array}{l}\text { Administradores, gerentes y di- } \\
\text { rectores }\end{array}$ & 67 & 3 & 6 & 76 & 321 \\
\hline Empleados de Oficina ............ & 53 & 5 & 0 & 58 & 602 \\
\hline 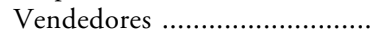 & 113 & 2 & 0 & 115 & 600 \\
\hline $\begin{array}{l}\text { Agricultores, pescadores y caza- } \\
\quad \text { dores }\end{array}$ & 1.307 & 48 & 1 & 1.356 & 4.184 \\
\hline Mineros y canteros .................... & 18 & 0 & 0 & 18 & 134 \\
\hline Transportes y Comunicaciones. & 53 & 0 & 0 & 53 & 348 \\
\hline $\begin{array}{l}\text { Artesanos y trabajadores afines .. } \\
\text { Servicios, deportes y diversio- }\end{array}$ & 420 & 6 & 0 & 426 & 2.761 \\
\hline 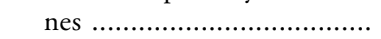 & 120 & 16 & 0 & 136 & 945 \\
\hline Fuerzas armadas ....................... & 51 & 0 & 0 & 51 & 279 \\
\hline $\begin{array}{l}\text { No consta, o no está bien espe- } \\
\text { cificado }\end{array}$ & 6.773 & 3.855 & 19 & 10.648 & 22.073 \\
\hline 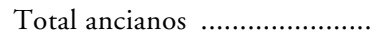 & 9.064 & 3.950 & 26 & 13.040 & \\
\hline Total población . .......................... & 23.440 & 9.392 & 67 & & 32.899 \\
\hline
\end{tabular}




\section{TABLA X}

Suicidios consumados y tentativas en la ancianidad (mayores de 60 años) por sexo y profesiones. Años 1976-1992

(Porcentajes)

\begin{tabular}{|c|c|c|c|c|c|}
\hline \multirow[b]{2}{*}{ Profesiones } & \multicolumn{3}{|c|}{ Sexo } & \multirow{2}{*}{$\begin{array}{l}\text { Total } \\
\text { más } 60 \text { años }\end{array}$} & \multirow{2}{*}{$\begin{array}{c}\text { Total } \\
\text { población }\end{array}$} \\
\hline & Hombres & Mujeres & No consta & & \\
\hline $\begin{array}{l}\text { Profesionales, técnicos y asimi- } \\
\quad \text { lados }\end{array}$ & 3,9 & 15,8 & 0,0 & 15,9 & 6,0 \\
\hline $\begin{array}{l}\text { Administradores, gerentes y di- } \\
\text { rectores }\end{array}$ & 2,9 & 3,2 & 85,7 & 23,7 & 2,9 \\
\hline Empleados de Oficina ............... & 2,3 & 5,3 & 0,0 & 9,6 & 5,6 \\
\hline Vendedores ................................. & 4,9 & 2,1 & 0,0 & 19,2 & 5,5 \\
\hline $\begin{array}{l}\text { Agricultores, pescadores y caza- } \\
\text { dores }\end{array}$ & 57,1 & 50,5 & 14,3 & 32,4 & 38,7 \\
\hline Mineros y canteros ................... & 0,8 & 0,0 & 0,0 & 13,4 & 1,2 \\
\hline Transportes y Comunicaciones . & 2,3 & 0,0 & 0,0 & 15,2 & 3,2 \\
\hline $\begin{array}{l}\text { Artesanos y trabajadores afines .. } \\
\text { Servicios, deportes y diversio- }\end{array}$ & 18,3 & 6,3 & 0,0 & 15,4 & 25,5 \\
\hline 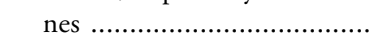 & 5,2 & 16,8 & 0,0 & 14,4 & 8,7 \\
\hline Fuerzas armadas .................... & 2,2 & 0,0 & 0,0 & 18,3 & 2,6 \\
\hline $\begin{array}{l}\text { Total en los que consta la pro- } \\
\text { fesión }\end{array}$ & 100,0 & 100,0 & 100,0 & 100,0 & 100,0 \\
\hline $\begin{array}{l}\text { Total en los que no consta y no } \\
\text { está bien especificado }\end{array}$ & 74,7 & 97,6 & 73,8 & 81,6 & 67,1 \\
\hline
\end{tabular}

conoce la actividad, los porcentajes más altos corresponden a los Agricultores, pescadores y cazadores (56,7 por 100) y Artesanos y trabajadores afines $(17,8$ por 100), lo cual es perfectamente lógico ya que, en esas edades, éstas son actividades que muchas personas (un 3,1 de la población anciana activa económicamente) siguen desarrollando después de rebasar la edad en la que otros (profesionales y trabajadores por cuenta ajena) se jubilan.

En todo caso, y viendo los datos relativos a los suicidios y tentativas en todas las edades, lo que sí parece apuntarse es que este fenómeno se da más en las actividades profesionales de menor cualificación: Agricultores, pescadores y cazadores $(38,6$ por 100$)$ y Artesanos y trabajadores afines (25,5 por 100). 


\section{REFLEXIONES SOBRE EL SUICIDIO EN LA ANCIANIDAD}

A nuestro juicio, de los tres grupos que establece Durkheim ${ }^{3}$ para la tipología de los suicidios — egoísta, altruista y anómico—- el correspondiente al suicidio en la ancianidad encajaría perfectamente en el tercero de los señalados, pues el anciano suicida llega a su determinación como consecuencia de la «anomia» en que se encuentra, de la desorganización vital que percibe dentro de su estructura social, de la solución de continuidad que se da en su espacio de comunicación intergeneracional y en relación, muchas veces directa, con el proceso de cambio social.

Quizá para ilustrar esta hipótesis baste señalar cómo mientras en el período 1883-1900, y según datos de C. Bernaldo de Quirós ${ }^{4}$, el índice de suicidios en personas mayores de 60 años era de 11,7 por 100, en el período 1970-1978 este mismo índice se elevaba al 44,5 por 100, y hasta 1986 se sitúa en el 40,1 por 100, y en 1992 lo tenemos en un 41,2 por 100. Es cierto que ha aumentado sensiblemente la proporción de personas ancianas en nuestra población, pero también es verdad que en el supuesto de coincidir exactamente todas las condiciones y circunstancias, independientemente de las cifras absolutas, las cifras relativas habrían de guardar una correlación.

En relación con este fenómeno del suicidio, que lo entendemos como un hecho sociopatológico, quisiéramos señalar, denunciándolo y proclamándolo, las dimensiones del mismo, intentando dar un aldabonazo a la conciencia de una sociedad en la que tan alta proporción de personas toman tan trágica decisión.

Los índices de suicidio aumentan con la edad, siendo más altos en los hombres que en las mujeres y, en las naciones con pluralismo racial, más elevado en los hombres blancos que en los de color.

Cuando se habla de suicidio infantil, las víctimas, ¿sabían o presentían que su decisión y sus actos iban a desembocar en un resultado irreversible? Esta y otras muchas interrogantes se nos presentan al tratar sobre el suicidio en los niños.

Salvo que el presunto suicida — niño, joven o adulto - hubiera dejado constancia expresa de las motivaciones de su intención, nos movemos siempre en el terreno de las conjeturas. James Toolan ${ }^{5}$ considera que las causas de suicidio o de intento de suicidio pueden agruparse, para los niños y adolescentes, en estas cinco categorías: $1 .^{\text {a) }}$ Enfado contra alguna persona, interiorizado en forma de culpa y depresión. 2. ${ }^{a}$ ) Intento de manipular o influir sobre ella, con

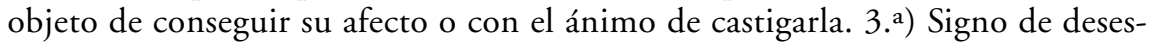
peración. 4. ${ }^{a}$ ) Reacción súbita ante la idea de desintegración interior. 5.a) Deseo de unirse a un pariente fallecido.

Estimamos que el suicidio infantil, en la mayor parte de los casos, no constituye un éxito en los propósitos, sino un fracaso, ya que la intencionalidad de

${ }^{3}$ E. Durkheim, El suicidio, Ed. Reus, S. A., Madrid, 1928.

${ }^{4}$ C. Bernaldo de Quirós, citado por M. Ruiz-Funes, en "Etiología del suicidio en España», en El suicidio, de E. DurkheIm, Ed. Reus, S. A., Madrid, 1928.

5 J. A. Toolan, "Suicide in children and adolescence», en Suicidal Behaviors, de Resnik, citado por P. Alcobendas, en Revista de la Juventud, núm. 28, 1965. 
buena parte de ellos es llamar la atención de alguna o algunas personas mayores, aunque no siempre, ya que un 70 por 100 de los niños que se quitan la vida ya han manifestado antes ideas de muerte y un 20 por 100 habían tenido previamente tentativas o tratan de huir de alguna amenaza o sanción real o imaginaria (temor a la actitud de los padres por unas calificaciones escolares deficientes, por ejemplo).

El suicidio o la tentativa en los grupos de edad más jóvenes se consigue o se intenta mediante el empleo, básicamente, de procedimientos más ambivalentes, como barbitúricos, analgésicos y somníferos, con lo que quizá, en lo más profundo del subconsciente o del infraconsciente, se está buscando y esperando que alguien llegue a tiempo de impedir que el resultado sea fatal e irreversible o, simplemente, están empleando unos métodos más acordes con la tecnología propia de su cultura urbano-industrializada. En los ancianos, los sistemas, métodos o medios empleados, como ya ha quedado expuesto, contrastan fuertemente con los anteriores, ya que mayoritariamente acuden a métodos violentos y definitivos como son la suspensión o asfixia (ahorcamiento), precipitación al vacío o al paso de vehículos (ferrocarril, tren metropolitano, etc.). Estos procedimientos no permiten dudar de su intencionalidad.

Los diferentes índices estudiados por Durkheim ${ }^{6}$ y J. M. de Miguel $^{7}$ entre las razas, los sexos, la edad, etc., así como la estimación de los procedimientos y métodos, indican que el ambiente social y los valores culturales y personales tienen una gran importancia, una importancia fundamental.

\section{MOTIVACIONES}

Por cuanto respecta a las motivaciones que pudieran impulsar a los ancianos al suicidio, creemos que entre las razones o causas principales cabría destacar la enfermedad, la debilidad física, el aislamiento, la soledad, como aparentemente desencadenantes de otras tales como el rechazo de los familiares, la sensación de estorbo, la inutilidad o la sensación de falta de cariño, muy acusada entre las personas ancianas, en virtud de su hipersensibilidad y de su tendencia a estar más pendientes de las formas que del fondo, en contraste con la actitud más superficial en las formas, pero que no tiene por qué implicar, forzosamente, menos profundidad de sentimientos en el fondo, de los más jóvenes, arrastrados por el ritmo de la vida y no tan pendientes de los detalles. En todo caso, para muchos ancianos estas sensaciones no son suposiciones ni imaginaciones, sino apreciaciones ciertas de una triste y lamentable realidad.

Pero para no quedarnos en las hipótesis y en las conjeturas, hemos examinado las causas conocidas de los suicidios de los ancianos en los años 1976 a 1992, que quedan detalladas en la tabla XI.

${ }^{6}$ E. Durkheim, op. cit.

7 J. M. De Miguel Rodríguez, El ritmo de la vida social, Ed. Tecnos, Madrid, 1973, pp. 274-356. 
TABLA XI

Causas de suicidio en la ancianidad, 60 y más años de edad

(Años 1976 a 1992)

\begin{tabular}{|c|c|c|c|c|}
\hline \multirow[b]{2}{*}{ Causas } & \multicolumn{3}{|c|}{ Sexo } & \multirow[b]{2}{*}{ Total } \\
\hline & Hombres & Mujeres & No consta & \\
\hline 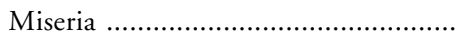 & 15 & 2 & 0 & 17 \\
\hline Pérdida de empleo ................................ & 7 & 0 & 0 & 7 \\
\hline 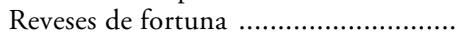 & 49 & 3 & 0 & 52 \\
\hline 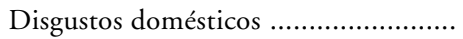 & 112 & 42 & 0 & 154 \\
\hline Amores contrariados ............................... & 8 & 2 & 0 & 10 \\
\hline 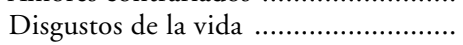 & 527 & 186 & 0 & 713 \\
\hline 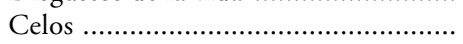 & 10 & 4 & 0 & 14 \\
\hline Temor a condena & 15 & 0 & 0 & 15 \\
\hline 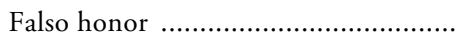 & 2 & 0 & 0 & 2 \\
\hline 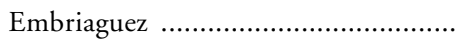 & 62 & 4 & 0 & 66 \\
\hline Padecimientos físicos ............................. & 2.052 & 665 & 4 & 2.721 \\
\hline Estado psicopatológico ....................... & 1.836 & 1.294 & 6 & 3.136 \\
\hline 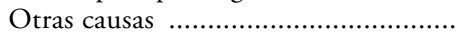 & 258 & 131 & 1 & 390 \\
\hline 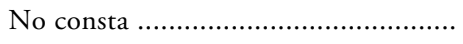 & 4.113 & 1.617 & 13 & 5.743 \\
\hline TOTAL & 9.066 & 3.950 & 24 & 13.040 \\
\hline
\end{tabular}

Independientemente de aquellos casos en que las causas se desconocen, que son la mayoría, en los que, de una u otra forma, se saben las causas, en su mayor proporción $(42,9$ por 100) corresponden a los trastornos psicopáticos, seguidos, por orden de importancia, por los padecimientos físicos (37,3 por 100 ), es decir, a falta de salud, a enfermedad física o psíquica. A una distancia significativa se encuentran los motivados por disgustos de la vida $(9,8$ por 100) y disgustos domésticos (2,1 por 100).

Hay que destacar que mientras que en las edades de la adolescencia los disgustos domésticos y de la vida afectan a más mujeres que hombres, en estas edades de la ancianidad es todo lo contrario. Entendemos que la razón estriba en que en las edades juveniles las chicas tienen un mayor sometimiento que los chicos a las directrices paternas o familiares, lo que provoca tales enfrentamientos y disgustos, mientras que en la ancianidad la mujer es más autosuficiente, aunque se encuentre viuda o sola, en tanto que los hombres que se hallan en estos estados suelen tener una dependencia mayor de sus hijos y familiares, viviendo en un hogar ajeno y padeciendo más las situaciones de controversia o de disgusto en familia, donde no siempre son o se sienten bien tratados.

También abundando en una idea manifestada con anterioridad, vemos cómo en las edades de la ancianidad el desequilibrio emocional, las tensiones psicológicas o el abatimiento ante la enfermedad están más acentuados en el hombre que 
en la mujer, la cual, además, posee una capacidad biológica superior, como lo prueba el hecho de la hipermortalidad masculina pues, pese a que nacen más varones que mujeres, mueren, desde las edades más tempranas, más hombres que mujeres. Para hacer tal aseveración nos basamos en el hecho de que, en los suicidios por estados psicopatológicos, un 58,5 por 100 son hombres, así como el 75,4 por 100 de los que lo han hecho por padecimientos físicos.

En cualquier caso, lo cierto es que la enfermedad o la falta de salud física o psíquica llevan a la autodestrucción a un 80,3 por 100 del total de los ancianos que, con causa conocida, llegan a este final. Por ello, consideramos oportuno hacer unas reflexiones sobre la interrelación de la salud física y la mental, toda vez que parece evidente que en unos casos es directamente la falta de salud física lo que lleva a tan considerable número de suicidios, y, por otra parte, cabe colegir que, en otro porcentaje también considerable, pueden ser precisamente las enfermedades físicas las que provoquen las perturbaciones mentales o los estados psicopatológicos que impulsen a otras personas a su fin.

El concepto de salud ha variado con el tiempo. En la antigüedad se definía simplemente como un buen estado del organismo: la utaxia, estado de perfecta salud de los griegos; la sanitas, calidad de sano de los romanos. En nuestro tiempo, popularmente, se define en forma negativa: no estar enfermo, sin síntoma o padecimiento alguno.

La Organización Mundial de la Salud (OMS), pensando en desterrar este concepto negativo, la definió como sigue: "La salud no significa tan sólo ausencia de enfermedad, sino un estado completo de bienestar físico, mental y social.»

Creemos que en el sector de la población que nos ocupa, la ancianidad, todos esos componentes tienen una trascendental importancia y se interrelacionan profundamente. De la salud física o de la salud social va a depender, mayormente, la salud mental. Y a través de este camino es por el que se puede llegar a la decisión final del suicidio como manifestación de conducta desviada y sociopatológica.

Modernamente, Matheney y Topalis definen la salud mental por hechos dependientes de las neuronas: «Es la capacidad de adaptarse a situaciones nuevas y resolver los problemas personales sin experimentar malestar intenso y conservando suficiente energía para ser un miembro constitutivo del bienestar colectivo de la sociedad.» Son, si cabe, aún más explícitas las definiciones de Johada y de From. La primera la define en función de seis atributos condensados en lo que sigue: conciencia y aceptación del propio yo, del desarrollo; modificación e integración de la propia personalidad, con sus corolarios de congruencia y de resistencia a las tensiones afectivas; autonomía, percepción recta de la realidad y dominio del medio personal para la solución de los problemas y para la acción eficaz. Según From, «el hombre mentalmente sano es el que usa la razón para aprehender objetivamente la realidad, el que sostiene con el mundo una relación amorosa, el que se siente como una individualidad singular sin dejar de sentir su solidaridad con el prójimo, el que no se somete a 
autoridades irracionales para acatar de buen grado la autoridad racional de la conciencia y de la razón, el que no deja de nacer mientras vive y considera la vida como el más precioso de los dones que ha recibido».

Para el anciano, la salud responde a las premisas antes señaladas: la física, la mental y la social, y nosotros nos atreveríamos a afirmar que la salud social ejerce en los ancianos de determinados sectores sociales, casi en la mayoría, tal presión que, efectivamente, son causa del origen del deterioro de la salud mental, en tanto que la falta de salud física provoca, a su vez, la carencia de la salud social en la medida en que ésta viene determinada por el comportamiento de la familia, en primera instancia, y del contexto social en general, en segundo término.

$\mathrm{Y}$, evidentemente, hemos de concluir que para determinados ancianos de nuestra sociedad que ya tienen su primer problema en el hecho de la adaptación a la vejez, que carecen en buena parte de servicios necesarios adecuados; para quienes sus problemas no son sólo de pensiones o de índole económica, con ser de una relevancia especial; que quisieran seguir trabajando muchos de ellos como consecuencia de esa falta de autonomía y de autosuficiencia económica, que algunos son abandonados por familiares ocasionalmente, que viven en soledad o aislamiento sin desearlo, que en cifras preocupantes - aunque, afortunadamente, cada vez más reducidas - disponen de asistencia médica y social limitada, el estado de su salud social es bastante precario. Es menester recordar que en la asistencia al anciano hay tres pilares fundamentales: servicios geriátricos hospitalarios, asistencia médica extrahospitalaria y servicios sociales de la comunidad, y que el fallo de cualquiera de estos tres pilares lleva consigo el fracaso del sistema asistencial.

El sociólogo analiza los datos, los expone, examina las causas y los efectos, trata de encontrar explicaciones a los hechos y, sin eludir competencias pero sin incurrir en el intrusismo ni en la asunción de cometidos que no son los suyos, los muestra a la sociedad. El sociólogo ha de tratar de responder, conforme afirma José Jiménez Blanco, a un determinado número de preguntas que se plantean desde la sociedad en relación con diferentes instituciones y estamentos que la componen. O porque, como dice Amando de Miguel, el sociólogo tiene que respirar las preocupaciones de su tiempo. Pero como ser humano y como miembro de un conjunto social concreto al que pertenece, profundamente preocupado por un tema como el que aquí hemos abordado, pretende que la conciencia social colectiva reciba un aldabonazo, confiando en que entre todos, familiares, educadores, psicólogos, políticos, sanitarios, sociólogos..., seamos capaces de poner los medios para reducirlo al máximo y tratar de conseguir la erradicación de este fenómeno que constituye una acusación a la calidad del entorno social y cuyas magnitudes son, evidentemente, alarmantes. 


\section{FUENTES}

Estadística de suicidio en España. Años 1976-80, INE, Madrid, 1985.

Estadística de suicidio en España. Años 1981-85, INE, Madrid, 1988.

Estadística de suicidio en España. Año 1986, INE, Madrid, 1988.

Estadística de suicidio en España. Año 1987, INE, Madrid, 1989.

Estadística de suicidio en España. Año 1988, INE, Madrid, 1989.

Estadística de suicidio en España. Año 1989, INE, Madrid, 1991.

Estadística de suicidio en España. Año 1990, INE, Madrid, 1991.

Estadística de suicidio en España. Año 1991, INE, Madrid, 1993.

Estadística de suicidio en España. Año 1992, INE, Madrid, 1994.

Movimiento Natural de la Población. Año 1976. Tomo III: Defunciones según la causa de muerte, INE, Madrid, 1979.

Movimiento Natural de la Población. Año 1977. Tomo III: Defunciones según la causa de muerte, INE, Madrid, 1980.

Movimiento Natural de la Población. Año 1978. Tomo III: Defunciones según la causa de muerte, INE, Madrid, 1981.

Movimiento Natural de la Población. Año 1979. Tomo III: Defunciones según la causa de muerte, INE, Madrid, 1983.

Movimiento Natural de la Población. Año 1980. Tomo III: Defunciones según la causa de muerte, INE, Madrid, 1986.

Movimiento Natural de la Población. Año 1981. Tomo III: Defunciones según la causa de muerte, INE, Madrid, 1987.

Movimiento Natural de la Población. Año 1982. Tomo III: Defunciones según la causa de muerte, INE, Madrid, 1987.

Movimiento Natural de la Población. Año 1983. Tomo III: Defunciones según la causa de muerte, INE, Madrid, 1988.

Movimiento Natural de la Población. Año 1984. Tomo III: Defunciones según la causa de muerte, INE, Madrid, 1988.

Movimiento Natural de la Población. Año 1985. Tomo III: Defunciones según la causa de muerte, INE, Madrid, 1989.

Movimiento Natural de la Población. Año 1986. Tomo III: Defunciones según la causa de muerte, INE, Madrid, 1990.

Movimiento Natural de la Población. Año 1987. Tomo I, INE, Madrid, 1991.

Movimiento Natural de la Población. Año 1988. Tomo I, INE, Madrid, 1992.

Movimiento Natural de la Población. Año 1989. Tomo I, INE, Madrid, 1992.

Movimiento Natural de la Población. Año 1990. Tomo I, INE, Madrid, 1993.

\section{BIBLIOGRAFIA}

Alcobendas Tirado, P. (1965): «El suicidio infantil y juvenil en España», Revista de la Juventud, núm. 28.

Arbelo Curbelo, A.; Hernández Rodríguez, G., y Arbelo lópez de letona, A. (1985): Demografía sanitaria de la adolescencia, Ministerio de Sanidad y Consumo, Madrid, pp. 9192 y $117-121$.

- (1986): Demografía sanitaria de la edad adulta, Ministerio de Sanidad y Consumo, Madrid, pp. 71-75.

- (1989): Demografia sanitaria de la ancianidad, Ministerio de Sanidad y Consumo, Madrid, pp. 283-296.

Bazo Royo, M. a T. (1989): «Personas ancianas: Salud y soledad», Revista Española de Investigaciones Sociológicas, núm. 47, pp. 193-223.

- (1990): La sociedad anciana, Ed. CIS/Siglo XXI, Col. Monografías, núm. 115, Madrid. 
Cobo Medina, C. (1982): «El suicidio infantil-juvenil en España», Tribuna Médica, núms. 933, 934 y 935,19 febrero a 5 marzo.

De Miguel Rodríguez, J. M. (1973): El ritmo de la vida social, Ed. Tecnos, Madrid, pp. 274356.

DurKheIM, E. (1928): El suicidio, Ed. Reus, S. A., Madrid.

HernÁNDEZ RodríGueZ, G. (1981): "Jubilación, aislamiento y suicidio en la tercera edad», en Libro de Actas del VIII Congreso Internacional de Universidades de Tercera Edad: El envejecimiento de la sociedad, su humanización y el compromiso con la tercera edad, Association Internationale des Universites du Troisième Age y Ministerio de Cultura de España, Madrid, 25-28 mayo.

- (1989): «Suicidio en la infancia y en la adolescencia», Tribuna Médica, núm. 1256, 24-30 noviembre.

Ruiz-Funes, M. (1928): «Etiología del suicidio en España», en El suicidio, Ed. Reus, S. A., Madrid.

Toolan, J. A.: «Suicide in children and adolescence», en Suicidal Behaviors, de Resnik, citado por P. Alcobendas en $o p$. cit.

\section{RESUMEN}

Desde 1970 a 1992 figuran como fallecidos por suicidio 46.917 personas, de las cuales el 41,78 por 100 contaban más de 60 años (Nomenclatura Internacional Detallada de causas de muerte). Se ha pasado de una media anual de 615 suicidios en la ancianidad desde 1970-1982 a 899 de 1983-1992. Los suicidios y tentativas en la ancianidad suponen en 1970 el 39 por 100 de los suicidios y tentativas en todas las edades, el 38 por 100 en 1976 y el 41 por 100 en 1992. En este trabajo se detallan y analizan los fallecimientos producidos por suicidio y se consideran también las tentativas en la ancianidad española, examinando el número de muertes acaecidas desde 1970 hasta 1992 por esta causa, su distribución por sexo (69,52 por 100 de hombres y 30,29 por 100 de mujeres), los métodos empleados (44,79 por 100 por ahorcamiento, 20,98 por 100 por precipitación desde lugar elevado, 14,16 por 100 por ahogamiento), las profesiones $(56,69$ por 100 son agricultores, ganaderos y pescadores) y las razones o causas conocidas que motivaron tal conducta. En el trabajo, al tiempo que se detallan, mediante las correspondientes tablas, los aspectos cuantitativos, se lleva a cabo una reflexión sobre el fenómeno estudiado y sus manifestaciones.

\section{ABSTRACT}

From 1970 to 1992, 46,917 people committed suicide (International Statistics on Death Causes-E950/E959) of whom 41.78\% were over 60 years old. From 1970 to 1982 the anual average was 615 suicides among the elderly per year. This anual average reached 899 suicides between 1983 and 1992. Suicides and attempted suicides in people aged over 60 years represented $39 \%$ of the total number of suicides in 1970 , 38\% in 1976 and $41 \%$ in 1992 . This study analyses deaths caused by suicide and attempted suicides of elderly people in Spain from 1970 to 1992. The research focuses on: the evolution of the number of deaths caused by suicide, their distribution by sex (69.52\% of men and $30.29 \%$ of women) and occupation $(56.69 \%$ farmers and fishermen...), the methods used to commit suicide ( $44.79 \%$ by hanging, $20.98 \%$ by jumping, $14.16 \%$ by drowning...), and the known causes of the suicide. The article includes the quantitative aspects of the issue concerned, as well as a reflection about the phenomenon studied and its manifestations. 